\title{
Performance of quantum dot nanobeads-based immunoassay in diagnosis of toxoplasmosis
}

\author{
Original Hamssa M Abd El-Ghani ${ }^{1}$, Nadia M Sabry ${ }^{1}$, Iman M Abdel-Salam ${ }^{1}$, Ibrahim R Ali ${ }^{2}$, \\ Article \\ Rasha F Mady ${ }^{3}$, Mona M Anwar ${ }^{1}$, Hala E Shams El-Din ${ }^{1}$
}

Departments of Medical Parasitology Faculty of Medicine, Ain Shams ${ }^{1}$ and Alexandria ${ }^{3}$

Universities, and Immunoparasitology and Evaluation of drugs, Theodor Bilharz Research Institute ${ }^{2}$, Egypt

\begin{abstract}
Background: Diagnosis of toxoplasmosis relies mainly on determination of IgG and IgM using ELISA. However, inter-laboratory evaluation of performance of many commercial kits gave unsatisfactory agreements. Merging the innovative use of quantum dots nanobeads (QDNBs) immunoassay may yield higher sensitivity and specificity than the conventional used methods.

Objective: The present study aims to evaluate the performance of QDNBs based immunoassay in serodiagnosis of human toxoplasmosis.

Subjects and Methods: The study included 175 subjects divided into three categories; 122 patients with clinical manifestations suggestive of toxoplasmosis; 32 patients proved positive for other parasitic infections, and 21 healthy control subjects. In comparison to Sabin Feldman dye test (SFDT), as a gold standard test, serum samples were examined in an immunoassay utilizing QDNBs for detection of T. gondii specific IgG and IgM antibodies.

Results: Compared to SFDT, combined QDNBs-IgG/IgM immunoassay exhibited sensitivity of 95.4\%, specificity of $98.9 \%$, positive predictive value (PPV) of 98.8\%, and negative predictive value (NPV) of $95.6 \%$, with relative agreement, validity, and accuracy of almost $97 \%$.

Conclusion: It was concluded that QDNBs-based immunoassay improves the performance of ELISA particularly sensitivity as well as shorten the assay time.
\end{abstract}

Keywords: diagnosis; human toxoplasmosis; immunoassay; quantum dots; T. gondii.

Received: 1 July, 2021, Accepted: 16 August, 2021.

Corresponding Author: Hala E. Shams El-Din, Tel.: +20 1005110726, E-mail: hala_elsayed80@yahoo.com

Print ISSN: 1687-7942, Online ISSN: 2090-2646, Vol. 14, No. 2, August, 2021.

\section{INTRODUCTION}

Toxoplasma gondii is an obligate intracellular protozoan that can infect any nucleated cell in nearly all warm-blooded animals, including human ${ }^{[1]}$. In Egypt, sero-prevalence rates of $68 \%$ and $26 \%$ have been recorded among pregnant females and meningoencephalitic patients, respectively ${ }^{[2]}$. Toxoplasmosis in neonates may result in severe neurological birth defects, including mental retardation and blindness ${ }^{[3]}$. Also, infection in immunocompromised patients can lead to serious complications as encephalitis, chorioretinitis, pneumonitis and disseminated infection with multiple-organ damage ${ }^{[4]}$.

Serodiagnosis by detection of specific antibodies remains the cornerstone for diagnosis of toxoplasmosis. However, serodiagnosis is confronted with many problems due to difficulty of interpretation of the tests' results and in determination of infection status; acute, chronic, congenital, reactivated. Sometimes, conventional assays may require complementary testing in reference laboratories where sophisticated techniques and experienced personals are available ${ }^{[5,6]}$. Moreover, false positive, negative, and discrepant results between different commercial kits were recorded ${ }^{[7-9]}$.

In an attempt to identify the interassay variability for quantification of anti-Toxoplasma IgG, no perfect correlation was found between any two of five immunoassays used ${ }^{[10]}$. Large differences in antiToxoplasma IgG titers were observed among the 5 immunoassays using serum samples from individual patients, from WHO international reference standards, and from standards and calibrators provided by the different five manufacturers. This highlights the importance of an accurate and reliable test for accurate diagnosis of toxoplasmosis, and hence the development of new serological assays.

Nanotechnology is of great use for medical diagnosis, and various nanoparticles (NPs) have exhibited tremendous potential for detecting disease markers $^{[11]}$. The convergence of nanotechnology and 
immunoassay has led to remarkable improvements in assay sensitivity and ease of operation ${ }^{[12]}$. Quantum dots (QDs) are NPs with a size of 2-10 $\mathrm{nm}$ and characterized by a large surface area to volume ratio, high loading capacity, facile synthesis, chemical stability, and ease of bioconjugation $^{[13]}$.

Aly et al. ${ }^{[14]}$ used QDNBs ELISA for diagnosis of cystic echinococcosis and recorded a sensitivity of $91.8 \%$, and a specificity of $88.8 \%$ in comparison to $85.7 \%$ and $84 \%$, respectively for traditional ELISA. Also, QDNBs based immunoassay was evaluated for detection of histidine rich protein-2 antigen for diagnosis of falciparum malaria and the results showed that the test allowed sensitive and quantitative measurement of the target antigen $^{[15]}$. In the same context, NPs were generally utilized in order to improve the serological diagnosis of toxoplasmosis; e.g., the use of gold nanoparticles (AuNPs) was reported several times in the development of new diagnostic tools for toxoplasmosis ${ }^{[16,17]}$. The aim of the present study is to evaluate the performance of QDNBs based immunoassay, as novel test, in serodiagnosis of human toxoplasmosis.

\section{MATERIAL AND METHODS}

This descriptive analytical study was conducted in Medical Parasitology Departments of Ain Shams, and Alexandria Universities, and Theodor Bilharz Research Institute, during the period from June 2018 to April 2021.

Study design: Blood samples were collected from patients with suggestive clinical manifestations of toxoplasmosis, and patients proved positive for other parasitic infections, as well as apparently healthy controls. Sera were examined using SFDT, and accordingly the study subjects were divided into five groups. Sera were also examined utilizing a prepared immunoassay composed of a probe formed of cadmium selenide/zinc sulfide (CdSe/ZnS)-QDNBs conjugated to goat polyclonal antihuman IgG and IgM-horseradish peroxidase (HRP) labeled conjugates for detection of T. gondii specific IgG and IgM antibodies, respectively. Sensitivity, specificity, PPV, NPV, relative agreements, validity, and accuracy of QDNBs were calculated in comparison to SFDT.

Study subjects: Blood samples (5 ml) were collected from inpatients and outpatients attending Ain Shams University Hospitals who had signs and symptoms suggestive of toxoplasmosis such as generalized and cervical lymphadenopathy, complicated pregnancies, delivery of infants with congenital malformations and immunocompromised patients. Blood samples were also collected from patients with collagen diseases and schizophrenic patients. This category of patients included 122 subjects. Study subjects also included patients with known other parasitic infections (32 control positive), as well as apparently healthy individuals (21 control negative). Sera were separated from all samples, aliquoted and labeled and kept frozen at $-20^{\circ} \mathrm{C}$ until tested.

Study groups: According to results of SFDT the patients and control subjects were classified into the following groups:

- Group 1a: Symptomatic patients with sera positive for Toxoplasma antibodies with titers $>1$ :16-<1:1024 suggestive of chronic infections.

- Group 1b: Symptomatic patients with serum positive for Toxoplasma antibodies with titers $\geq 1: 1024$ suggestive of acute infections.

- Group 2: Symptomatic patients with serum samples negative for Toxoplasma antibodies.

- Group 3: Negative for Toxoplasma antibodies and proved positive for other parasitic diseases by serology and/or direct parasitological methods (cystic echinococcosis, cryptosporidiosis, malaria, microsporidiosis, amoebiasis, giardiasis, urinary and intestinal schistosomiasis, and fascioliasis).

- Group 4: Apparently healthy non-symptomatic control subjects.

SFDT: All collected sera were first screened by SFDT ${ }^{[18]}$ with modifications according to Hoshina et al. ${ }^{[19]}$.

Sera examination for rheumatoid factor (RF): As RF is known to give false positive results in indirect IgMELISA assays, all sera were examined for RF by latex quick agglutination test (Avitex, Omega Diagnostics, UK). Positive samples were subjected to adsorption of RF using Serion RF adsorption kit according to the manufacturer specification (Serion Immunodiagnostica GmbH, Germany).

Synthesis and characterization of CdSe/ZnSQDNBs: CdSe QDs were prepared according to Xing et $a l^{[20]}$, followed by the shell formation step according to Dabbousi et al. and Xie et al. ${ }^{[21,22]}$, annealling at $260^{\circ} \mathrm{C}$ for $20 \mathrm{~min}$, then cooling to room temperature. Finally, core/shell QDs were isolated from solution with chloroform (Pubchem, USA) and methanol by centrifugation, washed three times in deionized water, and stored overnight in chloroform. Then the resulting NBs with polystyrene-co-maleic anhydride (PSMA) as matrix polymer were pelleted at $12,000 \mathrm{rpm}$ for $10 \mathrm{~min}$ and the supernatant was discarded. The pellet of NBs was washed three times in deionized water.

QDNBs were characterized by high resolution transmission electron microscope HR-TEM (JoelJem- 2100, Japan), Fourier-transform infrared (FTIR) spectroscope (Thermo fisher scientific, USA), powder X-Ray crystallography (Thermo fisher scientific, USA), Zeta potential analyzer (Jinan Winner Particle Instrument Stock Co., China) and Ultraviolet/ visible (UV/Vis) spectrophotometry (Schimadzu UV-1280, Japan). 
QDNBs-anti-human-IgG/IgM-HRP conjugates: Goat polyclonal antihuman IgG and antihuman IgM were conjugated to HRP using periodate method according to Tijssen and Kurstak ${ }^{[23]}$. This was followed by conjugation of anti-human(IgG/IgM) HRP conjugates to QDNBs according to Zhang et al. ${ }^{[24]}$. The antihuman IgG and IgM-HRP conjugates $(120 \mu \mathrm{g})$ were added to activated QDNBs and the mixture was incubated at room temperature for $2 \mathrm{~h}$. To confirm the binding of the QDNBs to HRP labeled antibodies, the fluorescence spectrum of QDNBs was measured before and after coupling with the antibodies.

Optimization of the immunoassay: Preliminary check board titrations were done to determine optimal antigen concentration, serum dilution and QDNBs antihuman-IgG and IgM-HRP conjugates dilution using antigen concentrations of $(1,5,10,15,20,25$ and 30) $\mu \mathrm{g} / \mathrm{ml}$, serum dilutions of $(1 / 25,1 / 50,1 / 100,1 / 200$, $1 / 400,1 / 600$ and $1 / 1200$ ) and QDNBs conjugates dilutions of $(1 / 250,1 / 500,1 / 1000,1 / 1500,1 / 2000$, $1 / 2500$ and $1 / 3000)^{[25]}$. Optical density (OD) signal developing time was detected after adding the QDNBs conjugates for $1 \mathrm{~h}$ and $30 \mathrm{~min}^{[26]}$. Different incubation time-periods with the o-phenylenediamine dihydrochloride (OPD) were tested for satisfactory development of the color reaction.

QDNBs-based immunoassay for detection of IgG and IgM antibodies in tested sera: The immunoassy was performed according to Gheshlaghchaei et al. ${ }^{[26]}$ and Ciaurriz et al. ${ }^{[27]}$ with QDNBs used instead of gold NBs. Antigen was prepared according to Hegazyet al., ${ }^{[28]}$. Antigen concentration, serum, and QDNBs conjugates dilutions and incubation times for each were performed according to results of preliminary check board titrations. Samples were tested in duplicates. The plates were read at $492 \mathrm{~nm}$ using ELISA reader and the results were expressed in the form of OD. Cutoff value for best discrimination between positive and negative results was determined by calculating mean and standard deviations for 18 Toxoplasma negative IgG and IgM serum samples. Samples that gave OD > mean \pm 2 SD were considered positive.
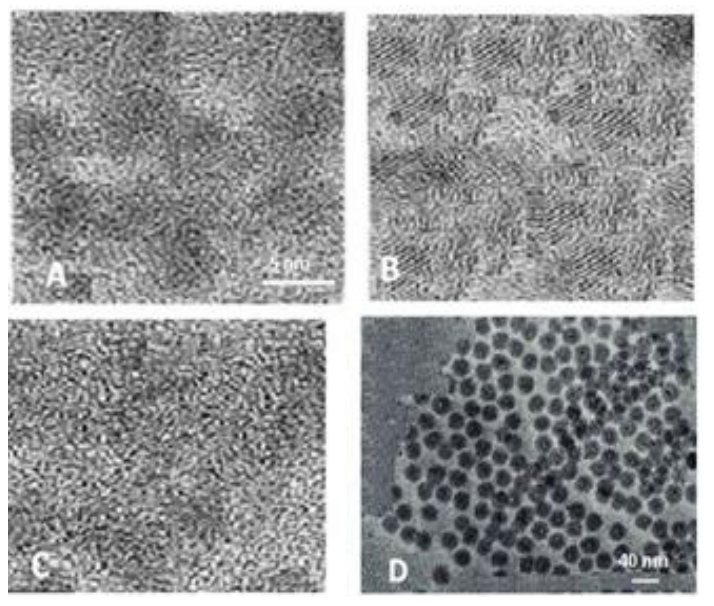
NB.
Statistical analysis: The collected data was revised, coded, tabulated and analyzed by the appropriate statistical tests using Statistical Package for Social Science (SPSS 20). Tests used included Chi-square test and Fisher's exact test. Sensitivity, specificity, PPV, NPV, validity and diagnostic accuracy were calculated for QDNBs immunoassay.

Ethical considerations: All subjects under the study were informed by the objective of the present work and consent was taken from each participant according to the regulations of the Egyptian Ministry of Higher Education and after having the required approval from the ethical committee of the Faculty of Medicine, Ain Shams University.

\section{RESULTS}

Characterization of CdSe/ZnS-QDNBs: Studying of $\mathrm{CdSe} / \mathrm{ZnS}$ by high resolution transmission electron microscopy (HR-TEM) showed that the prepared QDs were regular in shape, sufficiently mono distributed and well separated (Fig. 1 A-C). There was successful encapsulation of QDs into PSMA with estimated average size of about $60 \mathrm{~nm}$ and narrow size distribution (Fig. 1D).

The Fourier-transform infrared (FTIR) spectroscope analysis showed a prominent absorption band at 1702 $\pm 10 \mathrm{~cm}-1$ in CdSe/ZnS QDs that was due to the $\mathrm{C}=0$ stretch of carboxyl group and vibrations at 4257 and $4300 \mathrm{~cm}-1$ due to presence of hydroxyl $(-\mathrm{OH})$ group in $\mathrm{CdSe} / \mathrm{ZnS}$. This confirmed that QDs were water soluble and biocompatible (Fig. 2). The core/shell structure was identified by investigating the crystallographic properties by powder X-ray diffraction (XRD) (Fig 3). The zeta potential value was approximately $-30.2 \mathrm{mV}$ at peak and it was shifted after successful conjugation to polyclonal antihuman IgG/ IgM antibodiesHRP conjugates (Fig.4). Ultraviolet/visible (UV/ Vis) spectrophotometer was used to qualitatively characterize the successful conjugation of QDNBs to antihuman $\operatorname{IgG} / \operatorname{IgM}$ antibodies HRP conjugates and measure the concentration of QDNBs which was 2.33 $\mathrm{X} 10^{8}$ particles/ml as calculated by Beer's law (Fig.5).

Number of QDs in each NB was estimated as 501.9 QDs/bead and the number of HRP conjugated antibodies, per QDNBs (N) was approximately 37 HRPIgG antibodies/QDNB, and 33 HRP-IgM antibodies/ QDNB.

Fig. 1 (A-D). HR-TEM images of CdSe/ZnS-QDNBs. A-C show regularly shaped, sufficiently mono distributed and well separated QDs with a darker core surrounded by the shell. D shows successful encapsulation of QDs into polystyrene-co-maleic anhydride PSMA where QDs were dispersed in each 


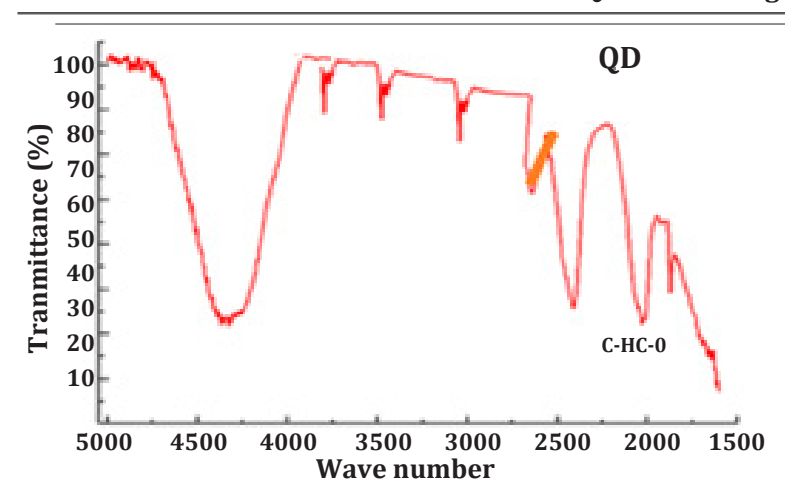

Fig. 2. Result of FTIR spectra analysis of QDNBs.

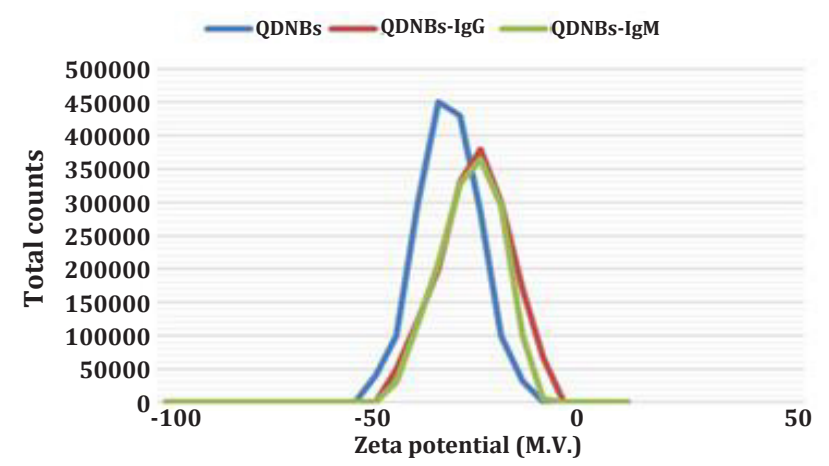

Fig. 4. Graph represents the zeta potential of CdSe/ZnSQDNBs (blue line), CdSe/ZnS-QDNBs/IgG (red line), and $\mathrm{CdSe} / \mathrm{ZnS}-\mathrm{QDNBs} / \mathrm{IgM}$ (green line).

Optimization of the immunoassay: The optimal antigen concentration, serum and conjugates dilutions were determined by preliminary check board titrations. It was observed that antigen concentration of $15 \mu \mathrm{g} / \mathrm{ml}$ coating buffer, serum dilution of $1 / 200$ in PBS-T and QDNBs-antihuman-IgG/IgM-HRP of $1 / 2000$ PBS-T were optimal for running the immunoassay. The optimal incubation time of QDNBs antihuman-IgG/IgMHRP conjugates with serum samples was 30 min. Five min were found to be optimal for the development of color reaction after the addition of the substrate. Cutoff value for positive samples were estimated for each run by calculating the mean ODs of 18 negative samples + 2 SD.

Inter-assay precision of QDNBs based immunoassay: This was assessed by running the same four serum Toxoplasma-IgG antibodies positive samples on four different days. They gave acceptable average coefficient of variation (CV\%) of $3.56 \%$.

Screening of patients and control subjects for antiToxoplasma antibodies by SFDT and RF by latex agglutination (Table 1): Out of 175 study and control subjects screened by SFDT, 87 (49.7\%) gave positive results. Of these, 27 patients (15.4\%) exhibited titers more than 1/1024, and 60 (34.3\%): 56 patients and 4 healthy control subjects, exhibited titers ranging from 1/16-1/256. The highest seropositivity was among patients complaining of lymphadenopathy

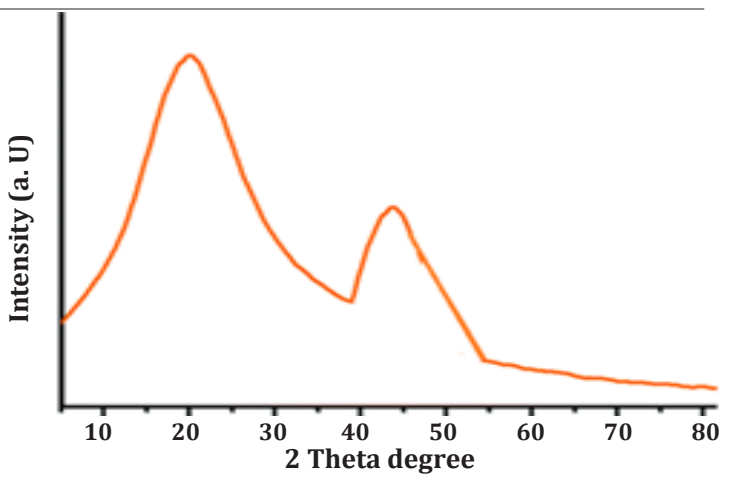

Fig. 3. X-ray powder diffraction patterns of prepared CdSe/ Zns-QDNBs.

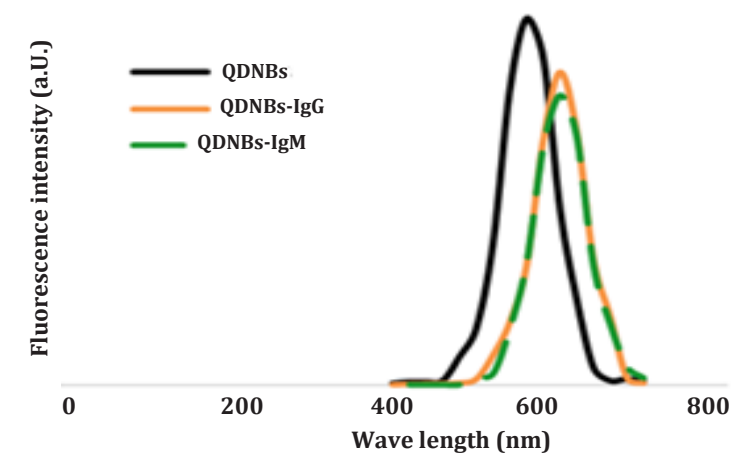

Fig. 5. Graph showing emission spectra of bare QDs (black line), QD-HRP-anti-IgG conjugates (orange line), and QDHRP-anti-IgM conjugates (dashed green line).

(81.8\%), followed by complicated pregnancies $(80 \%)$, schizophrenia (75\%), collagen diseases (69.6\%), congenital diseases (60.7\%), and immunosuppression $(44.4 \%)$. The only one case of chorioretinitis examined and 4 cases of the healthy control subjects reacted positively in SFDT with titers 1/16-1/128. Screening of the 175 sera for $\mathrm{RF}$, revealed that 41 (23.4\%) were seropositive mainly among patients with collagen diseases $(69.6 \%)$, followed by patients with schizophrenia (62.5\%), lymphadenopathy (45.5\%), complicated pregnancies (32\%), and immunosuppression (11.1\%).

Clinical presentation and demographic data of the groups under study (Table 2): The clinical presentation, age range and median, and gender of the patients and subjects belonging to the five groups under study were recorded.

Comparison of SFDT and QDNBs-IgG/IgM immunoassays (Table 3): For group 1a, out of 56 patients reacted positively in SFDT with titers ranging from 1/16-1/256, 52 (92.8\%) reacted positively in QDNBs-IgG immunoassay and none $(0 \%)$ gave positive reaction in QDNBs-IgM immunoassay. Out of the 27 patients of group $1 \mathrm{~b}$ who gave titers $\geq 1 / 1024$ in SFDT, $27(100 \%)$ and $19(70.4 \%)$ reacted positively in QDNBsIgG and QDNBs-IgM immunoassays, respectively. For group 2, out of the 39 symptomatic patients with negative SFDT, only $1(2.6 \%)$ gave positive reaction 
PARASITOLOGISTS UNITED JOURNAL

Table 1. Results of SFDT and rheumatoid factor screening for patients and control subjects.

\begin{tabular}{lcccccccc}
\hline \hline \multirow{2}{*}{\multicolumn{1}{c}{ Clinical presentation (No.) }} & SFDT & \multicolumn{4}{c}{ SFDT Titers } & \multicolumn{2}{c}{ RF } \\
\cline { 2 - 6 } & Positive & $\mathbf{1 / 1 6}$ & $\mathbf{1 / 3 2}$ & $\mathbf{1 / 6 4}$ & $\mathbf{1 / 1 2 8}$ & $\mathbf{1 / 2 5 6}$ & $>\mathbf{1 / 1 0 2 4}$ & Positive \\
\cline { 2 - 7 } & No (\%) & No (\%) & No (\%) & No (\%) & No (\%) & No (\%) & No (\%) & No (\%) \\
\hline Complicated pregnancies (25) & $20(80)$ & $2(8)$ & $1(4)$ & $1(4)$ & $0(0)$ & $0(0)$ & $16(64)$ & $8(32)$ \\
Congenital diseases (28) & $17(60.7)$ & $4(14.3)$ & $5(17.9)$ & $0(0)$ & $1(3.6)$ & $0(0)$ & $7(25)$ & $0(0)$ \\
Chorioretinitis (1) & $1(100)$ & $1(100)$ & $0(0)$ & $0(0)$ & $0(0)$ & $0(0)$ & $0(0)$ & $0(0)$ \\
Lymphadenopathy (11) & $9(81.8)$ & $1(9.1)$ & $0(0)$ & $3(27.3)$ & $1(9.1)$ & $2(18.2)$ & $2(18.2)$ & $5(45.5)$ \\
Immunosuppression (18) & $8(44.4)$ & $1(5.6)$ & $0(0)$ & $2(11.1)$ & $0(0)$ & $4(22.2)$ & $1(5.6)$ & $2(11.1)$ \\
Schizophrenia (16) & $12(75)$ & $4(25)$ & $2(12.5)$ & $3(18.8)$ & $2(12.5)$ & $1(6.25)$ & $0(0)$ & $10(62.5)$ \\
Collagen diseases (23) & $16(69.6)$ & $6(26.1)$ & $4(17.4)$ & $3(13.1)$ & $2(8.7)$ & $0(0)$ & $1(4.3)$ & $16(69.6)$ \\
Other parasitic infections (32) & $0(0)$ & $0(0)$ & $0(0)$ & $0(0)$ & $0(0)$ & $0(0)$ & $0(0)$ & $0(0)$ \\
Apparently healthy controls (21) & $4(19)$ & $2(9.5)$ & $0(0)$ & $1(4.8)$ & $1(4.8)$ & $0(0)$ & $0(0)$ & $0(0)$ \\
Total (175) & $87(49.7)$ & $21(12)$ & $12(6.9)$ & $13(7.4)$ & $6(3.4)$ & $8(4.6)$ & $27(15.4)$ & $41(23.4)$ \\
\hline \hline
\end{tabular}

SFDT: Sabin Feldman dye test; RF: Rheumatoid factor.

Table 2. Clinical presentation and demographic data of the groups under study.

\begin{tabular}{|c|c|c|c|c|c|}
\hline \multirow{3}{*}{$\begin{array}{l}\text { Groups } \\
\text { (No.) }\end{array}$} & \multirow{3}{*}{ Clinical presentations: No.(\%) } & \multicolumn{2}{|c|}{ Age } & \multicolumn{2}{|c|}{ Gender } \\
\hline & & Range & Mean \pm SD & Male & Female \\
\hline & & \multicolumn{2}{|c|}{ D: Day; M: Monthe; Y: Year } & No.(\%) & No.(\%) \\
\hline \multirow{7}{*}{$1 \mathrm{a}(56)$} & Complicated pregnancy: 4 (7.1) & $18-30 \mathrm{Y}$ & $21.5 \pm 4.4 Y$ & $0(0)$ & $4(7.1)$ \\
\hline & Congenital diseases: 10 (17.8) & $3 \mathrm{D}-1 \mathrm{Y}$ & $16 \mathrm{D}+0.28 \mathrm{Y}$ & $7(12.5)$ & $3(5.4)$ \\
\hline & Chorioretinitis: 1 (1.8) & $10 \mathrm{Y}$ & - & $0(0)$ & $1(1.8)$ \\
\hline & Lymphadenopathy: 7 (12.5) & $20-46 \mathrm{Y}$ & $36 \pm 8.23 \mathrm{Y}$ & $6(10.7)$ & $1(1.8)$ \\
\hline & Immunosuppression: 7 (12.5) & $18-47 \mathrm{Y}$ & $28+10.7 \mathrm{Y}$ & $1(1.8)$ & $6(10.7)$ \\
\hline & Schizophrenia: 12 (21.4) & $3-48 Y$ & $34.5 \pm 8.9 Y$ & $11(19.6)$ & $1(1.8)$ \\
\hline & Collagen diseases: $15(26.8)$ & $19-48 \mathrm{Y}$ & $32 \pm 8.3 \mathrm{Y}$ & $5(7.7)$ & $10(17.8)$ \\
\hline \multirow{5}{*}{ 1b (27) } & Complicated pregnancy: 16 (59.3) & $17-35 \mathrm{Y}$ & $24 \pm 5.4 Y$ & $0(0)$ & $16(59.3)$ \\
\hline & Congenital diseases: 7 (25.9) & $1 \mathrm{D}-5 \mathrm{~m}$ & $18 \pm 49.2 \mathrm{D}$ & $5(18.5)$ & $2(7.4)$ \\
\hline & Lymphadenopathy: 2 (7.4) & $47-53 \mathrm{Y}$ & $50 \pm 3 Y$ & $1(3.7)$ & $1(3.7)$ \\
\hline & Immunosuppression: 1 (3.7) & $20 Y$ & - & $0(0)$ & $1(3.7)$ \\
\hline & Collagen diseases: 1 (3.7) & $36 \mathrm{Y}$ & - & $0(0)$ & $1(3.7)$ \\
\hline \multirow{6}{*}{2 (39) } & Complicated pregnancy: 5 (12.8) & $20-39 \mathrm{Y}$ & $23 \pm 6.9 \mathrm{Y}$ & $0(0)$ & $5(12.8)$ \\
\hline & Congenital diseases: 11 (28.2) & $3 \mathrm{D}-6 \mathrm{Y}$ & $6 \mathrm{M} \pm 1.1 \mathrm{Y}$ & $5(12.8)$ & $6(15.3)$ \\
\hline & Lymphadenopathy: 2 (5.2) & $42-63 Y$ & $52 \pm 10 Y$ & $1(2.6)$ & $1(2.6)$ \\
\hline & Immunosuppression: $10(37.0)$ & $18-48 \mathrm{Y}$ & $41 \pm 9.6 \mathrm{Y}$ & $5(12.8)$ & $5(12.8)$ \\
\hline & Schizophrenia: 4 (10.4) & $17-34 \mathrm{Y}$ & $26 \pm 6 \mathrm{Y}$ & $2(5.1)$ & $2(5.1)$ \\
\hline & Collagen diseases: 7 (25.9) & $23-36 \mathrm{Y}$ & $27 \pm 4 Y$ & $0(0)$ & $7(17.9)$ \\
\hline \multirow{9}{*}{$3(32)$} & Cystic echinococcosis: 12 (37.5) & $29-65 \mathrm{Y}$ & $44 \pm 11 Y$ & $9(28.1)$ & $3(9.4)$ \\
\hline & Intestinal schistosomiasis: 5 (15.6) & $19-63 \mathrm{Y}$ & $49 \pm 14 Y$ & $4(12.5)$ & $1(3.1)$ \\
\hline & Urinary schistosomiasis: 2 (6.2) & $33-47 \mathrm{Y}$ & $40 \pm 7 \mathrm{Y}$ & $2(6.3)$ & $0(0)$ \\
\hline & Fascioliasis: 3 (9.4) & $29-45 \mathrm{Y}$ & $33 \pm 7 Y$ & $2(6.3)$ & $1(3.1)$ \\
\hline & Amoebiasis: 3 (9.4) & $35-65 \mathrm{Y}$ & $51 \pm 12 \mathrm{Y}$ & $1(3.1)$ & $2(6.3)$ \\
\hline & Cryptosporidiosis: 2 (6.2) & $23-36 \mathrm{Y}$ & $30 \pm 7 Y$ & $0(0)$ & $2(6.3)$ \\
\hline & Microsporidiosis: 2 (6.2) & $21-30 \mathrm{Y}$ & $26.5 \pm 5 Y$ & $2(6.3)$ & $0(0)$ \\
\hline & Malaria: $2(6.2)$ & $33-35 \mathrm{Y}$ & $34 \pm 1 Y$ & $2(6.3)$ & $0(0)$ \\
\hline & Giardiasis: 1 (3.1) & $20 \mathrm{Y}$ & - & $1(3.1)$ & $0(0)$ \\
\hline $4(21)$ & Apparently healthy control subjects & $21-67 \mathrm{Y}$ & $23 \pm 13.5 \mathrm{Y}$ & $7(33.3)$ & $14(66.7)$ \\
\hline
\end{tabular}


Table 3. Comparison between SFDT and QDNBs-immunoassay for detection of anti-Toxoplasma antibodies.

\begin{tabular}{lcccc}
\hline \hline \multirow{2}{*}{$\begin{array}{l}\text { Groups } \\
\text { (No.) }\end{array}$} & \multicolumn{2}{c}{ SFDT } & & \multicolumn{2}{c}{ QDNBs-immunoassay positive } \\
\cline { 2 - 5 } & Titer & No. (\%) & IgG & IgM \\
\hline $\mathbf{1 a}$ & $1 / 16$ & $22(39.3)$ & $20(35.7)$ & No. (\%) \\
& $1 / 32$ & $18(32.1)$ & $16(28.5)$ & $0(0)$ \\
& $1 / 64$ & $10(17.9)$ & $10(17.9)$ & $0(0)$ \\
& $1 / 128$ & $5(8.9)$ & $5(8.9)$ & $0(0)$ \\
& $1 / 256$ & $1(1.8)$ & $1(1.8)$ & $0(0)$ \\
$\mathbf{1 b}$ & $>1 / 1024$ & $27(100)$ & $27(100)$ & $19(70.4)$ \\
\hline $\mathbf{2}$ & $<1 / 16($ Negative) & $39(100)$ & $1(2.6)$ & $1(2.6)$ \\
\hline $\mathbf{3}$ & $<1 / 16($ Negative) & $32(100)$ & $0(0)$ & $0(0)$ \\
\hline $\mathbf{4}$ & $1 / 16$ & $2(9.5)$ & $2(9.5)$ & $0(0)$ \\
& $1 / 64$ & $1(4.8)$ & $1(4.8)$ & $0(0)$ \\
& $1 / 128$ & $1(4.8)$ & $1(4.8)$ & $0(0)$ \\
\hline \hline
\end{tabular}

1a: SFDT positive with titres $>: 16-<1: 1024$; 1b: SFDT positive with titres $>1: 1024 ; 2$ : SFDT negative; 3: Control positive; 4: Control negative.

in QDNBs-IgG and QDNBs-IgM immunoassays. Among the 32 patients of group 3 (control positive) with other serologically and parasitological confirmed parasitic infections and who gave negative reaction in SFDT, none $(0 \%)$ reacted positively in QDNBs IgG or IgM immunoassay, even with the 8 samples that had given positive reactions in IgM-parasite specific immunoassays. Only four subjects of the 21 (19.1\%) apparently healthy control negative subjects in group 4 , reacted positively in SFDT with titers of 1/16-1/128 and in QDNBs-IgG immunoassay, but not $(0 \%)$ in QDNBs-IgM immunoassay.

The agreements between SFDT and QDNBs IgG and IgM immunoassay are shown in tables (4 and 5), respectively. Significant agreements between SFDT and QDNBs immunoassays $(P<0.05)$ were found, although it was higher for QDNBs-IgG than QDNBs-IgM, with Kappa values of 0.94 and 0.76 , respectively.

Table 4. Agreement between SFDT and QDNBs based immunoassay in detection of T. gondii IgG antibodies.

\begin{tabular}{lcccc}
\hline \hline \multirow{2}{*}{ QDNBs IgG } & \multicolumn{3}{c}{ SFDT } & \multirow{2}{*}{ Agreement } \\
\cline { 2 - 4 } & Positive & Negative & Total & \\
\hline Positive & $83(47.43 \%)$ & $1(0.57 \%)$ & $84(48 \%)$ & Kappa: $\mathbf{0 . 9 4 3}$ \\
Negative & $4(2.29 \%)$ & $87(49.71 \%)$ & $91(52 \%)$ & $\boldsymbol{P}$ value $<\mathbf{0 . 0 0 1}$ \\
\hline Total & $87(49.71 \%)$ & $88(50.29 \%)$ & $175(100 \%)$ & Significant \\
\hline \hline
\end{tabular}

Table 5. Agreement between SFDT and QDNBs based immunoassay in detection of T. gondii IgM antibodies.

\begin{tabular}{lcccc}
\hline \multirow{2}{*}{ QDNBs IgM } & & SFDT & Agreement \\
\cline { 2 - 4 } & Positive & Negative & Total & Kappa: $\mathbf{0 . 7 6 3}$ \\
Positive & $19(15.96 \%)$ & $1(0.84 \%)$ & $20(16.81 \%)$ & P \\
Negative & $8(6.72 \%)$ & $91(76.47 \%)$ & $99(83.19 \%)$ & P value $<\mathbf{0 . 0 0 1}$ \\
\hline Total & $27(22.68 \%)$ & $92(77.32 \%)$ & $119(100 \%)$ & Significant \\
\hline \hline
\end{tabular}

QDNBs-IgG/IgM immunoassay diagnostic performance for detection of anti-Toxoplasma antibodies in comparison with SFDT (Table 6): The sensitivity specificity, PPV, NPV, relative agreement, validity, and accuracy of QDNBs combined IgG/IgM immunoassay in comparison with the SFDT gold standard test for serodiagnosis of toxoplasmosis were estimated. Combined QDNBs-IgG/IgM immunoassay exhibited sensitivity of $95.4 \%$, specificity of $98.9 \%$, PPV of $98.8 \%$ and NPV of $95.6 \%$.
Table 6. Diagnostic performance of combined QDNBs IgG/IgM immunoassay for diagnosis of toxoplasmosis in comparison with SFDT as gold standard.

\begin{tabular}{lc}
\hline \hline & $\begin{array}{c}\text { Combined QDNBs IgG/ } \\
\text { IgM immunoassay }\end{array}$ \\
\hline Sensitivity & $95.4 \%$ \\
Specificity & $98.9 \%$ \\
PPV & $98.8 \%$ \\
NPV & $95.6 \%$ \\
Relative agreement & $97.1 \%$ \\
Validity & $97.2 \%$ \\
Accuracy & $97.1 \%$ \\
\hline \hline
\end{tabular}




DISCUSSION

Serological diagnosis of toxoplasmosis and interpretation of the tests' results are confronted with many problems especially in diagnosis of acute infections in pregnant women, congenital infections in fetuses and newborns, infections in immunosuppressed patients and patients with chorioretinitis and collagen diseases ${ }^{[5,6,10,29]}$. ELISAs for detection of IgG or IgM are the most widely used tests for serodiagnosis of toxoplasmosis having several advantages including reproducibility, cost effectiveness, and flexibility in adapting desired research interests ${ }^{[30,31]}$. However, whether manual or automated, ELISAs are subjected to false negative results due to low levels of antibodies. Also false positive results, especially with IgM, may occur due to interference from RF, antinuclear antibodies, and other viral and bacterial diseases, or due to persistence of IgM from old infections ${ }^{[5,10,32]}$. Moreover, inter-laboratory evaluation of performance of many commercial kits for quantification of IgG antibodies gave unsatisfactory agreements ${ }^{[6,10]}$. In many instances, the results of ELISA should be confirmed in reference laboratories where other tests as SFDT, indirect fluorescent antibody test (IFAT), immunosorbent agglutination assays (ISAGA), serum IgG avidity test, and western blotting (WB) of serum from motherbaby pairs are available ${ }^{[5,6,10,29]}$; which would improve the diagnostic ability and reporting of the infection status. This necessitates the improvement of the sensitivity and specificity of routinely used ELISA.

The use of NPs in immunoassays may enhance the sensitivity, by increasing the number of enzyme molecules in the final antigen-antibody-enzyme complex. This will increase the catalysis of the substrate and lead to signal amplification in a single recognition reaction ${ }^{[12]}$. QDs are fluorescent inorganic semiconductor NPs with diameters in the range of 2-10 $\mathrm{nm}^{[33]}$ which can offer high luminescence with great long term colloidal stability and significant signal amplification. Also, the polymer matrix material facilitates the conjugation of biomolecules to the probes. Finally, by the encapsulation of several QDs into one NB as a single probe, the reporting signal can be directly amplified, without the need for additional signal enhancing steps ${ }^{[24]}$.

In the present study the diagnostic performance of CdSe/ZnS-QDNBs based immunoassay in serodiagnosis of human toxoplasmosis was studied to improve the efficiency of the present serological diagnostic methods. During optimization of the test, it was found that only 5 min incubation time with OPD substrate were needed to develop optimal color reaction. This fast response is apparently directly related to the increased concentration of enzyme activity in HRP-QDNB conjugate when used instead of HRP only. This agrees with the study of Ambrosi et $a l^{[34]}$ who showed that only a 5 min incubation period of the enzyme with 3,3',5,5'-tetramethylbenzidine was needed when a Au-nano bioconjugate was used, while in the conventional ELISA test about a $20 \mathrm{~min}$ incubation was necessary to develop the color. Also, Gheshlaghchaei et al. ${ }^{[26]}$ showed the same effect, where the time needed for the enzyme incubation with OPD substrate was reduced from 20 to 9 min for optimal color attainment, in comparison to traditional ELISA.

SFDT is the gold standard test for serodiagnosis of toxoplasmosis due to its high sensitivity. It is recommended as reference test for the confirmation of acute infection and for the validation of commercial kits $^{[35]}$. As SFDT detects whole IgG, IgM, IgA immunoglobulins, it permits very early diagnosis ${ }^{[36]}$; but the achieved antibody titers cannot accurately differentiate between acute or chronic infection ${ }^{[37]}$. In our study five groups classified on the basis of their SFDT results, were tested by QDNBs IgG/ IgM-immunoassay. The results showed significant agreement between SFDT and both IgG and IgM QDNBs immunoassays. The sensitivity of IgM immunoassay in detecting acute cases of group $1 \mathrm{~b}$ $(19 / 27 ; 70.4 \%)$ corresponds to $\geq 1: 1024$ titre in SFDT suggesting acute infection in immunocompetent patients, but doesn't confirm the presence of IgM antibodies $^{[38]}$. On the other hand, Remington et al. ${ }^{[29]}$ stated that SFDT detects mainly IgG antibodies, which may explain why, in the present study, the agreement was higher between SFDT and QDNBs-IgG than with QDNBs-IgM, with Kappa values of 0.94 and 0.76, respectively.

As recommended by Petersen et al. ${ }^{[39]}$, SFDT measures all immunoglobulin subclasses and therefore its results cannot be compared to those of isolated IgG or IgM assays, but only to combined IgG/IgM evaluation. In the present study the diagnostic performance of combined QDNBs-IgG/ IgM immunoassay compared with SFDT revealed sensitivity of $95.4 \%$, specificity of $98.9 \%$, PPV value of $98.8 \%$, NPV of $95.6 \%$, relative agreement, validity, and accuracy of almost $97 \%$. It is worth noting that none of the included sera from patients with other parasitic infections reacted positively in either QDNBs-IgG or IgM immunoassay denoting high specificity of the tests. Only four healthy control subjects gave positive reaction in QDNB-IgG which is expected as patients infected with Toxoplasma may appear healthy and are discovered by chance to have anti-Toxoplasma antibodies.

Additionally, QDs were used in immunoassays several times. Islam et $a l^{[40]}$ improved routine colorimetric detection for the measurement of aflibercept level (vascular endothelial growth factor) in plasma using a unique amplification reagent composed of QD-fluorescent beads conjugated to anti-goat antibodies. The beads offered high 
luminescence with relevant long-term colloidal stability and were able to improve sensitivity by 3 -fold ( $0.25 \mathrm{ng} / \mathrm{ml})$, and amplified signal significantly compared to conventional colorimetric methods. Aly et $a l^{[14]}$ evaluated QDNBs immunoassay for diagnosis of human cystic echinococcosis in human serum samples and recorded sensitivity of $91.8 \%$, and specificity of $88.8 \%$ in comparison to $75.5 \%$ and $60 \%$, respectively for traditional sandwich ELISA. Lv et al. ${ }^{[25]}$ used CdSe/ ZnS-QDNBs in quantitative detection of the C-reactive protein standard antigens by fluorescence linked immunoassay that requires half of the analysis time, and offers a lower limit of detection presenting a wide liner detection range than those of conventional ELISA.

Using anti-schistosomal monoclonal antibodies (MAb) loaded on AuNPs increased the sensitivity and specificity of sandwich ELISA for detection of circulating schistosomal antigen allowing easy detection of active and light infections ${ }^{[41]}$. Polystyrene NPs conjugated with polyclonal IgG antibodies specific for $P$. falciparum showed high sensitivity for the detection of P. falciparum antigen ${ }^{[42]}$. El-Shafey et $a l^{\left[{ }^{[3]}\right.}$ used anti-filarial antibodies IgG conjugated with AuNPs in nano-sandwich ELISA for detection of circulating filarial antigen in patients infected with $W$. bancrofti. They recorded higher sensitivity of $95.1 \%$, specificity of $87.5 \%$, PPV of $92.9 \%$, and NPV $91.3 \%$ in comparison with conventional ELISA that exhibited for the same parameters $90.7 \%, 75 \%, 84.8 \%$ and $84 \%$, respectively.

In the same context, NPs as well as QDs were generally used in order to improve the serological diagnosis of toxoplasmosis. For example, the use of AuNPs was reported in the development of new diagnostic tools for T. gondii infections. Al Hamshary et al. $^{[16]}$ evaluated the detection of Toxoplasma antigen (SAG1) in serum samples by nano-gold sandwich ELISA. The sensitivity and specificity were $89.2 \%$ and $94 \%$, respectively. In patients with other parasites only three cases were positive, two with G. lamblia and one with E. histolytica. Shaheen et al. ${ }^{[17]}$ evaluated the diagnostic performance of nano-gold beads-based ELISA in serodiagnosis of human toxoplasmosis based on detection of circulating Toxoplasma surface antigen (SAG3). The study showed that nano-gold ELISA had higher specificity, PPV, and diagnostic accuracy than conventional sandwich ELISA. While conventional ELISA gave higher sensitivity and NPV than nano-gold. Among 30 samples having other parasitic infections, only two patients with giardiasis gave positive reaction.

Jiang et $a l^{[44]}$ developed a dynamic flow immunochromatographic test (ICT) using AuNPs of $15 \mathrm{~nm}$ conjugated to staphylococcal protein A for the detection of Toxoplasma IgG antibodies in dogs and cats. The test showed high sensitivity and specificity of approximately $92 \%$ and $93.1 \%$, respectively, similar to the ELISA test. However, performance time for ICT was shorter than that for ELISA and required 10 to 20 times fewer samples versus conventional ICT. Aly et $a l^{[45]}$ used a nano sandwich ELISA based on bioconjugation of polyclonal antibody (pAb) IgG with Silica nanoparticles $\left(\mathrm{SiO}_{2}\right)$ for detection of circulating Toxoplasma lysate antigen in sera and urine of patients suspected of infection with T. gondii and found that the sensitivity of the assay was $90.0 \%$. All the negative controls were below the cutoff value while 4 out of 30 patients with other parasites infections were at the borderline of the cutoff value giving $92.0 \%$ specificity. False positive cases belonged to the group of patients infected with Cryptosporidium spp. and G. lamblia. Medawar-Aguilar et al. ${ }^{[46]}$ developed and applied a laser-induced fluorescence immnunosensor, based on the integration of chitosan zinc oxide NPs into the microfluidic channel, to quantify anti- $T$. gondii IgG antibodies. It significantly improved the sensitivity of the method and simplified the operation process decreasing the analysis time. Hegazy et al. ${ }^{[25]}$ studied immunomagnetic bead-ELISA using SAG1 for diagnosis of human toxoplasmosis and showed sensitivity, specificity, PPV, and NPV of 98\%, 96.4\%, $96 \%$, and $98.1 \%$, respectively. While $2 / 35$ samples from individuals infected with other parasites (one with E. histolytica and one with Cryptosporidium spp.) were borderline. Li et $a .^{[47]}$ developed a lateral flow immunochromatographic assay (LFIA) for detection of specific IgM to causative agents of TORCH infections during pregnancy (toxoplasmosis, rubella, cytomegalovirus, and herpes simplex virus infections). This LFIA using goldmagnetic NPs modified with polymath acrylic acid, and conjugated with antihuman IgM antibodies, obtained $100 \%$ sensitivity and specificity, plus the ability to simultaneously detect multiple pathogens.

In conclusion, QDNBs have large surface area-tovolume ratio that allow the attachment of multiple enzyme-conjugated antibodies which generates an amplified colorimetric signal compared with a single enzyme-conjugated antibody. Therefore, QDNBs apparently improve the performance of ELISA particularly sensitivity as well as shorten the assay time.

Acknowledgment: The authors are grateful to Prof. Khalifa E. khalifa, Medical Parasitology Department, Faculty of Medicine, Ain Shams University, for his critical reviewing and sharing in writing and revising the manuscript.

Author contribution: Abd El-Ghani HM shared in preparation of QDs and performance of the practical experiments and wrote the manuscript. Sabry NM and Abdel Salam IM shared in planning, designing, and supervising the research. Ali I supervised the practical work of coating QDs and performing the immunoassay. Mady RF supervised the practical work of SFDT. Anwar MM and Shams El-Din HA shared in writing and revising the manuscript. 
Conflict of interest: The authors declare that they have no conflict of interest.

Funding statement: This research did not receive any specific grant from funding agencies in the public, commercial, or not-for-profit sectors.

\section{REFERENCES}

1. Nabi H, Rashid I, Ahmad N, Durrani A, Akbar H, Islam S, et al. Induction of specific humoral immune response in mice immunized with ROP18 nanospheres from Toxoplasma gondii. Parasitol Res 2017; 116:359-370.

2. Helmy YA, El-Adawy $\mathrm{H}$, Abdelwhab EM. A comprehensive review of common bacterial, parasitic and viral zoonoses at the human-animal interface in Egypt. Pathogens 2017; 6:33-50.

3. Hampton MM. Congenital toxoplasmosis: A review. Neonatal Netw 2015; 34(5):274-278.

4. Wang ZD, Liu HH, Ma ZX, Ma HY, Li ZY, Yang ZB, et al. Toxoplasma gondii infection in immunocompromised patients: A systematic review and meta-analysis. Front microbiol 2017; 8: 389.

5. Azab ME, Khalifa KE. Review of problems confronted in the serodiagnosis of toxoplasmosis. Egypt J Lab Med 1996; 8(3):707- 728.

6. Dard C, Fricker-Hidalgo H, Brenier-Pinchart MP, Pelloux H. Relevance of and new developments in serology for toxoplasmosis. Trends Parasitol 2016; 32(6):492-506.

7. Leslé F, Touafek F, Fekkar A, Mazier D, Paris L. Discrepancies between a new highly sensitive Toxoplasma gondii ELISA assay and other reagents: Interest of Toxoplasma IgG western blot. Eur J Clin Microbiol Infect Dis 2011; 30(10):1207-1212.

8. Zhang K, Lin G, Han Y, Li J. The standardization of 5 immunoassays for anti-Toxoplasma immunoglobulin G (IgG). Clin Chim Acta 2017; 472:20-25.

9. Simon L, Fillaux J, Guigon A, Lavergne RA, Villard O, Villena I, et al. Serological diagnosis of Toxoplasma gondii: analysis of false-positive IgG results and implications. Parasite 2020; 27:1-7.

10. Zhang K, Lin G, Han Y, Li J. Serological diagnosis of toxoplasmosis and standardization. Clin Chim Acta 2016; 461:83-89.

11. Sato-Berru R, Saniger JM, Flores JO, Sánchez-Espindola M. Simple method for the controlled growth of SiO2 spheres. J Mater Sci Eng 2013; A3:237-242.

12. Gao Y, Zhou Y, Chandrawati R. Metal and metal oxide nanoparticles to enhance the performance of enzymelinked immunosorbent assay (ELISA). ACS Applied Nano Mater 2020; 3(1):1-21.

13. Banerjee A, Pons T, Lequeux N, Dubertret B. Quantum dots-DNA bioconjugates: synthesis to applications. Interface Focus 2016; 6(6):20160064.

14. Aly IR, El-Sayed H, Hamad RS, Elenin G. Evaluation of quantum dots nanobeads based immunoassay for diagnosis of human hydatidosis. ISER International Conference, Kuala Lumpur, Malaysia 2018.
15. Kim C, Hoffmann G, Searson PC. Integrated magnetic bead-quantum dot immunoassay for malaria detection. ACS Sens 2017; 2(6):766-772.

16.AL-Hamshary AS, Bayoumi IR, Aly NS, Omar RE, Mohammed DA, Marei YM, et al. Evaluation of nano-based-ELISA for serodiagnosis of human toxoplasmosis. Benha J Applied Sciences 2020; 5:1-7.

17. Shaheen H, Abdelmonem W, Baiuomy I, El-Ahl S. Evaluation of the diagnostic performance of nano gold beads-based ELISA for detection of circulating Toxoplasma surface antigen 3 (SAG3) in serum samples of infected cases. JRAM 2020; 1 : 2090-7265.

18. Sabin AB, Feldman HA. Dyes as microchemical indicators of a new immunity phenomenon affecting a protozoon parasite, Toxoplasma. Science 1948; 108 (2815):660-663.

19. Hoshina T, Fukumoto S, Aonuma H, Saiki E, Hori S, Kanuka H. Seroprevalence of Toxoplasma gondii in wild sika deer in Japan. Parasitol Int 2019; 71:7679.

20.Xing B, Li W, Dou H, Zhang P, Sun K. Systematic study of the properties of CdSe quantum dots synthesized in paraffin liquid with potential application in multiplexed bioas says. J Phys Chem C 2008; 112(37):14318-14323.

21. Dabbousi BO, Rodriguez-Viejo J, Mikulec FV, Heine JR, Mattoussi H, Ober R, et al. (CdSe) ZnS core-shell quantum dots: synthesis and characterization of a size series of highly luminescent nanocrystallite. J Phys Chem C1997; 101 (46): 9463-9475.

22. Xie R, Kolb U, Li J, Basché T, Mews A. Synthesis and characterization of highly luminescent CdSe core CdS/Zn0.5Cd0.5S/ZnS multi-shell nanocrystals. J Am Chem Soc 2005; 127(20):7480-7488.

23. Tijssen P, Kurstak E. Highly efficient and simple methods for the preparation of peroxidase and active peroxidase-antibody conjugates for enzyme immunoassays. Anal Biochem 1984; 136(2):451457.

24. Zhang P, Lu H, Chen J, Han H, Ma W. Simple and sensitive detection of HBs Ag by using a quantum dots nanobeads based dot-blot immunoassay. Theranostics 2014; 4(3): 307- 315.

25. Lv Y, Wu R, Feng K,, Li J, Mao Q, Yuan H, et al. Highly sensitive and accurate detection of C-reactive protein by $\mathrm{CdSe} / \mathrm{ZnS}$ quantum dotbased fluorescence-linked immunosorbent assay. J Nanobiotechnol 2017; 15:15-35.

26. Gheshlaghchaei I, Madani S, Golchinfar R, Emami F, Gholami TH. Development of a nano-ELISA system for the rapid and sensitive detection of H9N2 avian influenza. Arc Razi Institute 2016; 71(1):29-34.

27. Ciaurriz P, Fernández F, Tellechea E, Moran JF, Asensio AC, Beilstein J. Comparison of four functionalization methods of gold nanoparticles for enhancing the enzyme-linked immunosorbent assay (ELISA). Nanotechnol; 2017; 8:244-253. 
28. Hegazy S, Farid A, Rabae I, El-Amir A. Novel IMB-ELISA assay for rapid diagnosis of human toxoplasmosis using SAG1 antigen. Jpn J Infect Dis 2015; 68(6):474-480.

29. Remington JS, Thulliez P, Montoya JG. Recent developments for diagnosis of toxoplasmosis. J Clin Microbiol 2004; 42(3):941-945.

30. Rostami A, Karanis P, Fallahi S. Advances in serological, imaging techniques and molecular diagnosis of Toxoplasma gondii infection. Infection 2018; 46(3):303-315.

31. Liyanage KL, Wiethoelter A, Hufschmid J, Jabbar A. Descriptive comparison of ELISAs for the detection of Toxoplasma gondii antibodies in animals: A systematic review. Pathogens 2021; 10(5):605.

32. Murata FHA, Previato M, Frederico FB, Barbosa AP, Nakashima F, de Faria GM Jr, et al. Evaluation of serological and molecular tests used for the identification of Toxoplasma gondii infection in patients treated in an ophthalmology clinic of a public health service in São Paulo State, Brazil. Front Cell Infect Microbiol 2020; 9: 472.

33. Cui L, He X, Chen G.Recent progress in quantum dot based sensors. RSC Adv 2015; 5:26644-26653.

34. Ambrosi A, Airò F, Merkoçi A. Enhanced gold nanoparticle based ELISA for a breast cancer biomarker. Anal Chem 2010; 82(3):1151-1156.

35. Kasper DC, Prusa AR, Hayde M, Gerstl N, Pollak A, Herkner KR, et al. Evaluation of the vitros ECiQ immunodiagnostic system for detection of antiToxoplasma immunoglobulin $\mathrm{G}$ and immunoglobulin $\mathrm{M}$ antibodies for confirmatory testing for acute Toxoplasma gondii infection in pregnant women. J Clin Microbiol 2009; 47(1):164-67.

36. Reiter-Owona I, Petersen E, Joynson D, Aspöck H, Dardé M L, Disko R, et al. The past and present role of the Sabin-Feldman dye test in the serodiagnosis of toxoplasmosis. Bulletin of the World Health Organization 1999; 77(11): 929-935.

37. Ybañez R, Ybañez AP, Nishikawa Y. Review on the current trends of toxoplasmosis serodiagnosis in humans. Front Cell Infect Microbiol 2020; 10:204.

38. Asai T, Mizuno F, Kojima S, Takeuchi T, Kobayashi A, Suzuki Y. High correlation in antibody titers between the Sabin-Feldman dye test and an enzyme-linked immunosorbent assay detecting immunoglobulin G antibodies to the nucleoside triphosphate hydrolase of Toxoplasma gondii. J Clin Microbiol 1992; 30(5):1291-1293.

39. Petersen E, Borobio M, Guy E, Liesenfeld O, Meroni V, Naessens A, Spranzi E, et al. European multicenter study of the LIAISON automated diagnostic system for determination of Toxoplasma gondii-specific immunoglobulin G (IgG) and IgM and the IgG avidity index. JCM 2005; 43: 1570-1574.

40. Islam R, Chitforoushzadeh Z, Kar S, Aikey R, McEwen K. Evaluation of an ELISA method using novel amplification reagent and its comparison with traditional colorimetric ELISA assay for the measurement of aflibercept in biological matrices. Pharm Sci 2018; 360:50-55.

41. Kamel M, El-Baz H, Demerdash Z, El-Karaksy S, ElGendy N, Hassan S, et al. Nano-immunoassay for diagnosis of active schistosomal infection. World J Medical Sci 2016; 13 (1):27-37.

42. Thiramanas R, Jangpatarapongsa K, Asawapirom U, Tangboriboonrat P, Polpanich D. Sensitivity and specificity of PS/AA-modified nanoparticles used in malaria detection. MicrobBiotechnol 2013; 6:406413.

43. El-shafey OK, Afifi AF, Oshiba SF, Aly IR, Lasheen ME. Evaluation of gold nanoparticles coated sandwich ELISA as innovative diagnosis for human bancroftian filariasis . JESP 2018; 48(1):223-232.

44. Jiang $\mathrm{W}$, Liu $\mathrm{Y}$, Chen $\mathrm{Y}$, Yang $\mathrm{Q}$, Chun $\mathrm{P}$, Yao $\mathrm{K}$, et al. A novel dynamic flow immunochromatographic test (DFICT) using gold nanoparticles for the serological detection of Toxoplasma gondii infection in dogs and cats. Biosens Bioelectron 2015; 72:133-139.

45. Aly I, Taher EE, El Naina G, El Sayed H, Mohammed FA, Hamad RS, et al. Advantages of bioconjugated silica-coated nanoparticles as an innovative diagnosis for human toxoplasmosis. Acta Tropica 2018; 177:19-24.

46. Medawar-Aguilar V, Jofré C, Fernández Baldo $M$, Alonso A, Angel S, Raba J et al. Serological diagnosis of toxoplasmosis disease using a fluorescent immunosensor with chitosan-ZnO-nanoparticles. Anal Biochem 2018; 564:116-122.

47. Li X, Zhang Q, Hou P, Chen M, Hui W, Vermorken A et al. Gold magnetic nanoparticle conjugate-based lateral flow assay for the detection of IgM class antibodies related to TORCH infections. Int J Mol Med 2015; 36:1319-1326. 